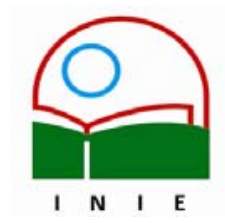

Universidad de Costa Rica

Facultad de Educación

Instituto de Investigación en Educación

ACTUALIDADES INVESTIGATIVAS EN EDUCACION

\title{
LA CALIDAD UNIVERSITARIA DESDE UNA PERSPECTIVA DE GÉNERO
}

\section{Carolina Bolaños Cubero ${ }^{1}$}

Resumen: El artículo plantea la necesidad de que la evaluación de carreras e instituciones, en el marco del discurso de la calidad universitaria y la rendición de cuentas, considere criterios que valoren las condiciones curriculares en que hombres y mujeres se relacionan durante el desarrollo de una carrera. Además de cómo estas relaciones contribuyen o no a modificar, las formas tradicionalmente discriminatorias en perjuicio de las mujeres. Se ofrece una serie de elementos que pueden derivar en criterios de evaluación, desde una perspectiva de género y que, necesariamente, contribuyen a definir la calidad universitaria.

Palabras clave: CALIDAD UNIVERSITARIA/ PERSPECTIVA DE GÉNERO/ CRITERIOS DE CALIDAD/

Abstract: El artículo plantea la necesidad de que la evaluación de carreras e instituciones, en el marco del discurso de la calidad universitaria y la rendición de cuentas, considere criterios que valoren las condiciones curriculares en que hombres y mujeres se relacionan durante el desarrollo de una carrera. Además de cómo estas relaciones contribuyen o no a modificar las formas tradicionalmente discriminatorias en perjuicio de las mujeres. Se ofrece una serie elementos que pueden derivar en criterios de evaluación desde una perspectiva de género y que necesariamente contribuyen a definir la calidad universitaria.

Key words: HIGHER EDUCATION QUALITY/ GENDER OUTLOOK/ QUALITY CRITERIAV

\section{INTRODUCCIÓN}

La calidad universitaria ha venido siendo tema de interés, tanto del ámbito académico como político, desde ya hace varios años. Por ello, los discursos acerca de la calidad de la formación universitaria y de las instituciones de educación superior, apelan a la necesidad de realizar procesos de evaluación del quehacer universitario en forma constante, de modo que pueda garantizarse a la sociedad una adecuada formación de profesionales y un eficiente uso de los recursos, sobre todo cuando de fondos y servicios públicos se trata. ${ }^{2}$

\footnotetext{
${ }^{1}$ Licenciada en la Enseñanza de la Química de la Universidad de Costa Rica. Actualmente se desempeña como docente-investigadora en el Departamento de Investigación y Evaluación del Centro de Evaluación Académica, Universidad de Costa Rica.
}

Correo electrónico:

Artículo recibido: 14 de julio, 2005

Aprobado: 29 de setiembre, 2005

2 Para comprender más el surgimiento de la rendición de cuentas y el discurso de la calidad universitaria, puede verse el artículo "Acreditación: calidad o instrumento de legitimación para la Educación Superior", de Elsiana Guido, incluido en este mismo número. 
La evaluación se ha planteado entonces, como el mecanismo por el cual las universidades rinden cuentas y garantizan calidad. Como cualquier proceso evaluativo se requieren parámetros que orienten y definan las valoraciones y los juicios de esa calidad. Por tanto, con el fin de definir parámetros de calidad comunes, se han establecido criterios de valoración que contemplan diferentes dimensiones y aspectos curriculares y organizacionales de las instituciones de educación superior. Cada universidad decide bajo qué parámetros de calidad quiere valorarse o, por lo menos, selecciona aquellos- ya definidos por otros -que se ajustan más a sus intereses, necesidades o demandas.

La universidad tiene entonces que observar lo que la sociedad requiere y debe satisfacer estas necesidades, por medio de la formación de profesionales idóneos, así como del desarrollo de proyectos de investigación y de acción social pertinentes.

La sociedad es en sí misma dinámica. Esto lo podemos constatar al realizar un análisis socio-histórico de cualquier ámbito. Por ejemplo, desde el punto de vista tecnológico, existe una gran diferencia entre las posibilidades que tienen las generaciones jóvenes actuales, con las que tuvimos las ya no tan jóvenes y las que tuvieron las más viejas $\mathrm{y}$, más aún, con las generaciones que nos precedieron. $\mathrm{Y}$ esto sin remontarnos siglos atrás, sino solamente unas dos o tres generaciones. En el campo de las actividades económicas, ha habido grandes transformaciones, desde el tipo de actividades que se realizan, hasta quienes participan en dichas actividades. Por ejemplo, hoy hombres y mujeres participan en estas actividades, de manera insospechada un siglo atrás. Las crisis económicas y el descubrimiento de la productividad femenina, han introducido a las mujeres en las actividades económicas y productivas $y$, con ello, se ha generado la necesidad de cualificar su fuerza de trabajo, por lo que el acceso a la educación por parte de las mujeres, ha ido en aumento significativo.

Este dinamismo de la sociedad, requiere y demanda de las universidades también un permanente proceso de transformación y dinamismo.

En el caso de las mujeres, el acceso al trabajo fuera del hogar y su profesionalización, no ha implicado, sin embargo, un cambio sustantivo en la estructura social patriarcal; y las universidades, por su parte, no han promovido las transformaciones necesarias en este sentido. Por lo tanto, las universidades han contribuido a la reproducción de roles 
estereotipados y al mantenimiento de dicha estructura social. Como lo plantea Dennis García de la Universidad Central del Ecuador, al referirse a los hallazgos del proyecto Educación Alternativa con visión de género,

[...] la educación superior reproduce concepciones androcéntricas, [...] los contenidos y prácticas educativas mantienen en muchos aspectos un carácter sexista en docentes y estudiantes; que un limitado grupo de mujeres profesionales acceden a la universidad como docentes, que la universidad no orienta a las estudiantes a optar por carreras no tradicionales, consagrando la desvalorización de las carreras "femeninas". (Universidad Central del Ecuador, 2000, p.13)

Es por esta razón, que las universidades como las estatales costarricenses (y centroamericanas), tienen una gran responsabilidad en promover los cambios que la sociedad necesita, para el logro del bien común, de una verdadera justicia social, del desarrollo integral y de la libertad e independencia plena de nuestros pueblos, como reza el Estatuto Orgánico de la Universidad de Costa Rica, en su artículo 3.

De esta manera, el compromiso de las universidades para procurar la igualdad entre los géneros y promover una sociedad justa, libre de la opresión de género (entre otras) y de la violencia, es hoy una gran tarea, que muchas universidades ya han empezado a desarrollar. Las universidades latinoamericanas han desarrollado proyectos y esfuerzos importantes para evidenciar la discriminación de que son objeto las mujeres en nuestra sociedad y en las mismas universidades, así como para modificar las prácticas reproductoras del sistema patriarcal.

Sin ningún interés por enumerarlas todas, lo cual sería tedioso e innecesario, sino con el afán de ofrecer una pincelada de muestra de diferentes acciones, tenemos, por ejemplo, una propuesta sobre la Promoción de la Equidad de género en la Curricula de las Ciencias de la Salud (Vergés y Name, 2002); las Demandas de las mujeres universitarias (Isch y Romero, 2001). En Costa Rica debemos señalar el trabajo realizado por el Centro de Investigación en Estudios de la Mujer de la Universidad de Costa Rica; así como las acciones desarrolladas por la Universidad Nacional, la Universidad Estatal a Distancia y el Instituto Tecnológico de Costa Rica, algunas de ellas en estrecha colaboración entre estas 
universidades estatales ${ }^{3}$. Además de las acciones, la producción académica en este ámbito, también ha sido fructífera, como las desarrolladas en la UNAM de México, entre las que tenemos Cultura universitaria y género: los valores profesionales en la Facultad de Letras. El caso del Colegio de Pedagogía (Bedolla y otros, s.f.). Algunas producciones nacionales como Roles de género y mujeres académicas (Fernández, 2000 II); así como el titulado Currículum universitario género sensitivo e inclusivo (Bolaños, 2003).

\section{ACCIONES EN LA UNIVERSIDAD DE COSTA RICA}

En la Universidad de Costa Rica, ha habido diversas acciones que, desde hace años se han venido desarrollando y que, al articularse, han fundado el Centro de Investigación en Estudios de la Mujer (CIEM), en el desarrollo de la Maestría en Estudios de Género en conjunto con la Universidad Nacional. Así como el establecimiento de normativas como la referida al uso del lenguaje inclusivo en todos los documentos oficiales y producidos en la universidad y el Reglamento en contra del hostigamiento sexual.

EI CIEM, además de acoger los proyectos de investigación, que académicos y académicas plantean en temas de género y estudios de la mujer, vela por el cumplimiento de la normativa respectiva y por el desarrollo de acciones que contribuyan a que la comunidad universitaria, se sensibilice respecto a las relaciones de género, así como en brindar procesos de capacitación.

Estableciendo convenios con otras instituciones, se realizan proyectos de envergadura nacional e internacional. En este marco de cooperación internacional, se ha desarrollado, en la Universidad de Costa Rica el Programa de Acción Social PREVENCIÓN DE LA VIOLENCIA EN CONTRA LAS MUJERES EN COSTA RICA, con la cooperación de la Universidad de Toronto, Canadá. Este programa incluye el proyecto de currículo inclusivo y sensible a las diferencias de género, en el cual participan varias unidades académicas de la UCR, desde el año 2002 y hasta el 2006.

\footnotetext{
${ }^{3}$ Entre estas tenemos el caso de la Maestría en Estudios de la Mujer que desarrollan conjuntamente la Universidad de Costa Rica y la Universidad Nacional; así como la participación de la Universidad Estatal a Distancia en proyectos del CIEM.
} 
Como parte de este proyecto de currículo inclusivo y sensible a las diferencias de género, el Centro de Evaluación Académica (CEA) realiza un diagnóstico de la curricula universitaria desde una perspectiva de género, que posibilite la identificación de criterios de calidad que puedan orientar, tanto los procesos de desarrollo curricular, como los de evaluación de carreras. A este centro le compete coordinar los procesos evaluativos de las carreras que ejecutan las unidades académicas, en el marco de la calidad universitaria y la rendición de cuentas.

\section{DIAGNÓSTICO SOBRE LA INCLUSIVIDAD Y LA SENSIBILIDAD A LAS DIFERENCIAS DE GÉNERO EN LA CURRICULA UNIVERSITARIA.}

El proyecto Currículo universitario inclusivo y sensible a las diferencias de género, consta de varios sub-proyectos que están siendo desarrollados por varias instancias universitarias. Una de ellas es el Centro de Evaluación Académica, que realiza un diagnóstico de la inclusividad y la sensibilidad a las diferencias de género de la curricula universitaria. Con este diagnóstico se pretende construir un panorama de las prácticas universitarias, lo cual posibilite la determinación de criterios de calidad con perspectiva de género para ser considerados en los procesos evaluativos de las carreras universitarias, así como también en los procesos de diseño curricular (Bolaños, C.; Quirós, M; 2004).

El estudio consta de dos fases complementarias: 1) una consulta a la comunidad universitaria, por medio de cuestionarios para estudiantes, docentes, personal administrativo y autoridades; y 2) el análisis de una muestra de planes de estudio y de documentos utilizados en el CEA para las asesorías curriculares y evaluativos. Hasta el momento se ha realizado y analizado la consulta a docentes y estudiantes, y se está en el proceso de revisión y análisis de los documentos, a partir de guías elaboradas para tal fin, para concluir con el informe final en agosto del 2006.

\section{LA PERSPECTIVA DE GÉNERO}

La concepción del mundo que ha imperado, implica una determinada representación de los géneros, los estereotipos sociales y las normas que rigen la convivencia entre hombres y mujeres. Todo esto interviene en la configuración de la subjetividad de cada persona y de la cultura. Esta cosmovisión no es estática ni única, a lo largo de la vida de las personas y en las sociedades pueden existir simultáneamente varias cosmovisiones $\mathrm{y}$, sobre todo, estas pueden cambiar. 
La concepción androcéntrica que ha dominado el mundo, parte del principio fundamental de que la diferencia entre hombres y mujeres - natural (y hasta de naturaleza divina)- implica la supremacía de un género en relación con el otro, concepción que deriva del referente positivista que arrastramos desde el siglo XVIII. De ahí que se haya desarrollado una sociedad patriarcal, en la que el género masculino ha dominado y oprimido al género femenino, negando para este último, las oportunidades que han tenido los hombres. Por tanto, esta sociedad se caracteriza por la negación de la existencia de las mujeres en todos los ámbitos de la vida, quedando su accionar, socialmente aceptado, limitado solamente a algunos aspectos, especialmente en cuanto a la reproducción.

Surge entonces, la necesidad imperante de construir una sociedad en la que hombres y mujeres convivamos en ambientes de mutuo respeto y en condiciones equitativas en todos los ámbitos, de construir una humanidad diversa y democrática (Lagarde, 1997, p. 13).

A pesar de lo arraigado y de la diversidad de mecanismos de dominación que se han desarrollado en el patriarcado, el cambio es posible, y las mujeres de diversas regiones se han organizado para evidenciar las inequidades y las injusticias, y han luchado por crear otra cosmovisión de género y con ella una transformación de la sociedad. Este proceso ha implicado la construcción de teoría y metodologías, para la explicación y la comprensión de lo que ocurre a las mujeres y a los hombres $\mathrm{y}$, con ello, de la manera de entender los conceptos que intervienen en las relaciones entre los géneros.

A esta forma científica, crítica y analítica se le denomina perspectiva de género y como nos lo plantea Lagarde, “..., se conoce como perspectiva de género a esta visión científica, analítica y política creada desde el feminismo." (1997, p.15). Para comprender la perspectiva de género, es importante ubicarla epistemológicamente.

Al respecto la misma autora nos ilustra que, desde el punto de vista teórico, la perspectiva de género se basa en el paradigma histórico-crítico, y desde el punto de vista cultural en el feminismo.

Esta perspectiva reconoce la diversidad de géneros y la existencia de las mujeres y los hombres, como un principio esencial en la construcción de una humanidad diversa y democrática. Sin embargo, plantea que la dominación de género produce la opresión de género y ambas obstaculizan esa posibilidad. Una humanidad diversa y 
democrática requiere que mujeres y hombres seamos diferentes de quienes hemos sido, para ser reconocidos en la diversidad y vivir en la democracia genérica. (Lagarde, 1997, p.13).

Así, el propósito fundamental de desarrollar un currículo inclusivo y sensible a las diferencias de género, es decir un currículo diseñado y desarrollado desde una perspectiva de género, es posibilitar, desde la academia, desde la formación profesional, desde la convivencia en las aulas y fuera de éstas, el cambio en las relaciones entre los géneros y la construcción de esa humanidad diversa y democrática.

\section{PROPÓSITOS DEL DIAGNÓSTICO}

Así entendida la perspectiva de género $y$, con el afán de contribuir desde los procesos curriculares de diseño y evaluación en la consecución de la sociedad justa, equitativa, diversa y democrática, el CEA se ha planteado en el diagnóstico los siguientes propósitos.

1. Identificar acciones discriminatorias en contra de las mujeres que se presentan en la administración, la docencia y la investigación en la sede universitaria Rodrigo Facio.

2. Desarrollar estrategias que posibiliten la eliminación de la discriminación por género en las diferentes actividades académicas que se realizan en la Universidad de Costa Rica.

\section{CONSULTA REALIZADA}

El estudio consiste en un diagnóstico con perspectiva de género de la curricula universitaria, en el que participan estudiantes, docentes, personal administrativo, decanatos y direcciones académicas de la Universidad de Costa Rica.

Constituye el primer diagnóstico institucional acerca de la inclusividad y la sensibilidad a las diferencias de género en la administración, docencia e investigación universitarias, que se espera aporte información para diseñar y poner en ejecución estrategias para la sensibilización de la comunidad universitaria en la aplicación de la perspectiva de género en la Universidad de Costa Rica. 
El estudio contempla, en primera instancia, una consulta a diferentes poblaciones, en la que se interroga a las personas participantes acerca de aspectos de la vida universitaria, en términos de la existencia o no de acciones discriminatorias en la forma en la que se llevan a cabo las diferentes actividades e interacciones en la Universidad de Costa Rica. Como se indicó anteriormente, además de la consulta, el estudio incluye el análisis de documentos oficiales que se utilizan para orientar los procesos de diseño y evaluación curricular; así como el análisis de una muestra de planes de estudio de carreras que se imparten en la Universidad de Costa Rica. Se pretende que al poder contar con el diagnóstico, desde esta perspectiva, sea posible buscar y proponer en forma conjunta acciones para la eliminación de cualquier forma de discriminación de las mujeres en nuestra institución.

Los aspectos que se señalan en el presente artículo, corresponden a los aprendizajes que hasta ahora se han tenido y que se derivan de la primera parte del estudio, es decir, de la consulta a la población universitaria en la Sede Rodrigo Facio.

\section{ASPECTOS CONSIDERADOS}

El siguiente cuadro indica las poblaciones consideradas para la consulta y los aspectos indagados en cada una de ellas.

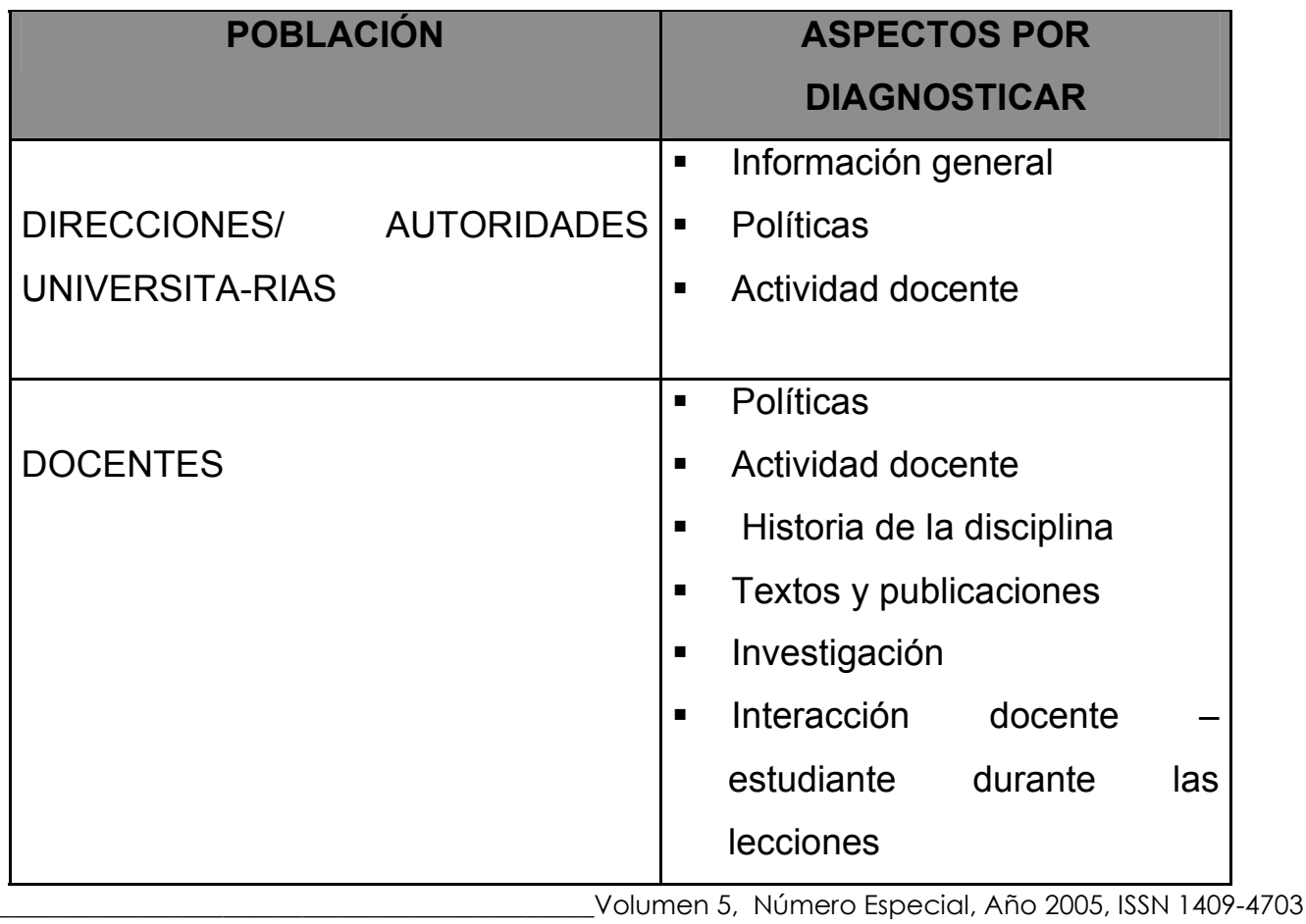




\begin{tabular}{|c|c|}
\hline ESTUDIANTES & $\begin{array}{l}\text { - } \text { Actividad docente } \\
\text { - Historia de la disciplina } \\
\text { - } \text { Textos y publicaciones } \\
\text { - Investigación } \\
\text { - Interacción docente - } \\
\text { estudiante durante las } \\
\text { lecciones }\end{array}$ \\
\hline PERSONAL ADMINISTRATIVO & $\begin{array}{ll}\text { - } & \text { Información general } \\
\text { - } & \text { Actividad docente } \\
\text { (contextualizada) }\end{array}$ \\
\hline
\end{tabular}

Fuente: Elaboración propia con base en la propuesta metodológica del diagnóstico.

Cada uno de los aspectos definidos para consultar a cada población, se desglosó en una serie de elementos que dieron origen a las preguntas que conformaron el cuestionario respectivo. Estos elementos y preguntas correspondientes, constituyen la base para la derivación de criterios de calidad con perspectiva de género.

\section{DERIVACIÓN DE CRITERIOS}

De estos aspectos considerados en la consulta, será necesario determinar y priorizar aquellos por considerar en la definición de criterios de calidad, desde una perspectiva de género en los procesos evaluativos. Para esta definición y priorización, será necesario desarrollar, desde las instancias pertinentes (CEA, CIEM y otras) procesos de diálogo y negociación en la comunidad universitaria.

Las acciones irían entonces en el sentido de hacer evidente lo que permanece oculto y potenciar la solución de muchos de los problemas durante los mismos procesos evaluativos, así como por medio de las acciones regulatorias que se planteen como consecuencia de la evaluación.

Por el momento, lo que intento aquí es presentar los aspectos considerados en el diagnóstico y que pueden constituir una primera base para la definición de criterios de 
calidad desde una perspectiva de género. Se presentan de acuerdo con cada uno de los aspectos indicado en el cuadro anterior.

\section{POLÍTICAS}

- Equilibrio en la proporción entre estudiantes hombres y estudiantes mujeres. Equilibrio en la proporción entre docentes hombres y mujeres en propiedad.

- Prevención y atención de comportamientos sexistas del personal administrativo y docente hacia las estudiantes.

- Prevención y atención de comportamientos sexistas entre estudiantes.

- Prevención y atención de comportamientos heterosexistas y homofóbicos en el personal administrativo y docente y en el estudiantado.

- Prevención del hostigamiento sexual contra estudiantes y personal docente y administrativo.

\section{ACTIVIDAD DOCENTE}

- Atención y trato que reciben hombres y mujeres estudiantes, por parte de los o las docentes cuando reciben clase.

- Concepción que tienen docentes y estudiantes acerca de la capacidad intelectual de hombres y mujeres

- Preconcepciones que tienen las profesoras y los profesores en relación con la orientación sexual de alumnos y alumnas. (Por ejemplo, dar por sentado que la población estudiantil es heterosexual.)

- Actitudes de los estudiantes hombres hacia las estudiantes mujeres.

- Oportunidades que tienen hombres y mujeres de la carrera de conseguir trabajo después de su graduación.

\section{PLAN DE ESTUDIO}

- Inclusión en la mayoría de los cursos de temas, problemas y ejemplos de interés específico para las mujeres o que se relacionan con lo que ellas viven o experimentan. 
- Empleo de textos que recurren a ilustraciones con contenido o ejemplos y texto que tratan en forma igualitaria a hombres y mujeres.

- Empleo de textos que recurren a ilustraciones, ejemplos o texto con contenido sexista, racista o xenofóbico.

- Inclusión de cursos en los que se enseña Teoría de Género.

- Inclusión de cursos en los que se aplica perspectiva de género como enfoque para analizar la historia, situaciones de la realidad, teorías y problemas, entre otros.

- Inclusión de experiencias, actividades, problemas y aportes de las mujeres como objeto de cursos separados.

- Enfoques teóricos que se enseñan y su relación con la promoción de prejuicios sexistas, racistas, homofóbicos o xenofóbicos.

- Aprendizaje y comprensión de las relaciones de género, por parte de la población estudiantil, como resultado de los cursos obligatorios y optativos

- Incremento de la comprensión de los aportes de las mujeres al desarrollo científico, económico, político, cultural y/o social, como resultado de los cursos obligatorios y optativos

- Aprendizaje por parte de estudiantes mujeres y hombres sobre los roles de género de hombres y mujeres, como resultado de los cursos obligatorios y optativos.

- Aprendizaje por parte de estudiantes mujeres y hombres sobre las desigualdades, discriminación y violencia de género hacia las mujeres en diversos ámbitos de la disciplina y la sociedad, como resultado de los cursos obligatorios y optativos.

IV. ABORDAJE DE LA HISTORIA DE LA DISCIPLINA (en cursos e investigaciones)

- Forma en la que es tratada la historia de la disciplina, tanto escrita como transmitida en cursos. (Enfatiza hechos y acontecimientos, realizaciones y avances científicos significativos en los que dominan los hombres o hay ausencia de mujeres, por ejemplo.)

- Forma en la que son tratadas las mujeres en la historia de la disciplina (por ejemplo incorpora solamente unas cuantas mujeres famosas, generalmente presentadas como "atípicas" o meritorias por logros o contribuciones esperadas solo de varones). 
V. TEXTOS, LECTURAS Y PUBLICACIONES (empleadas en los cursos y las investigaciones)

- Utilización de literatura en la que las mujeres son autoras o coautoras de los textos, publicaciones e investigaciones.

- Citas de mujeres como referencias en los textos, materiales educativos, informes de investigación y otros documentos producidos por la Unidad Académica.

- Formato para escribir referencias bibliográficas (es posible identificar el sexo de las personas autoras).

- En imágenes representadas en fotos incorporadas en los textos, investigaciones y otros materiales producidos por la unidad académica; están igualmente representados hombres y mujeres; como en posiciones de poder o mando.

- Forma en la que son incluidas las mujeres en los textos, investigaciones y otros materiales consultados y producidos por la unidad académica: como tema en el índice temático, como grupos de mujeres particulares, en secciones separadas sobre mujeres; como mujeres excepcionales, como problema, patología o en situación de crisis o víctima.

- En los textos, materiales e investigaciones producidas por la unidad académica, son tratados los problemas, experiencias o condiciones de mujeres y hombres, por separado, sin compararles.

- Los problemas, experiencias o condiciones de las mujeres son descritas / analizadas tanto separada como comparativamente con las de los hombres, buscando enfatizar en sus interrelaciones, impactos diferenciados e implicaciones.

- Percepción que tienen los académicos y las académicas acerca de si es posible o no incluir capítulos o secciones separadas o artículos dedicados a las mujeres.

\section{RELACIONES ENTRE DOCENTES Y ESTUDIANTES DURANTE LAS LECCIONES}

- Atención y escucha que da el y la docente a todas y todos los estudiantes cuando hablan.

- Forma en la que plantea preguntas a los hombres y a las mujeres (difíciles a los hombres y fáciles a las mujeres, p.e.). 
- Retroalimentación y estímulo que da a las mujeres y a los hombres cuando comentan o responden en clase y en su trabajo escrito. (de manera igualitaria o se parcializa).

- Tendencia, por sexo, para seleccionar asistentes o para dar información sobre oportunidades profesionales como conferencias e investigaciones.

- Prejuicios y estereotipos de los y las docentes respecto a mujeres y hombres. (Por ejemplo circunscribe ciertas cuestiones como el cuidado de la familia como "cuestiones de mujeres").

- $\quad$ Tipo de chistes que utiliza el y la docente (Por ejemplo, sobre cuestiones asociadas a las mujeres como igualdad salarial o violencia doméstica).

- Tendencia, por sexo, en la interrupción que hace a los y las estudiantes cuando exponen o hacen observaciones. (Por ejemplo, interrumpe a las estudiantes mujeres más a menudo que a los varones).

- $\quad$ Frecuencia con la que dirige preguntas en clase a hombres y mujeres estudiantes.

- Empleo de humor sexual o analogía erótica.

- Actitud que tiene ante el trabajo de mujeres investigadoras o autoras. (Ejemplo ridiculización o denigración).

- $\quad$ Actitud que tiene cuando estudiantes hombres asumen comportamientos denigrantes hacia las mujeres, las interrumpen, o usan conductas que limitan su participación en clase.

- $\quad$ Tipo de cumplidos que utiliza para hombre y mujeres. (Ejemplo, alaba a las mujeres por su apariencia y a los hombres por su trabajo).

\section{INVESTIGACIÓN}

- Uso del lenguaje "masculino" como genérico (Por ejemplo hombre, individuo, sujeto, niños, adultos cuando nos referimos tanto a hombres como a mujeres).

- Utilización de la experiencia masculina o problemas que interesan o afectan a hombres como la norma.

- Comparación de las mujeres en función de lo que ocurre a los hombres, lo que les preocupa a éstos, sus comportamientos.

- Existencia de una sobre valoración de lo masculino, de lo que hacen los hombres y cómo lo hacen, de sus problemas, intereses y demandas. 
- Neutralidad de género. Asumen que hombres y mujeres se ven afectados de la misma manera frente a una determinada situación y contexto.

- Tratan o evalúan distinto situaciones que son esencialmente idénticas, tomando como base el sexo de la persona o personas involucradas. (Por ejemplo, se tiende a tratar el embarazo en mujeres adolescentes con mayor dureza y crítica que en los hombres adolescentes).

\section{CONCLUSIÓN}

En la concepción de la calidad universitaria por tanto, habrá de considerarse también criterios que garanticen la igualdad de oportunidades y condiciones entre los géneros de modo que a la par de una formación profesional científica, un currículo bien estructurado, una adecuada biblioteca, sistemas de información eficientes, una administración honesta y comprometida con los recursos públicos, también se considere la calidad de las relaciones relaciones entre hombre y mujeres durante el proceso de formación y, su impacto en la construcción de una sociedad justa, igualitaria y libre de prejuicios y estereotipos, en particular en cuanto a las relaciones de género se refiere.

Lo planteado aquí ha pretendido esbozar algunos aspectos que pueden referir ¿cómo una universidad puede promover la equidad e igualdad entre los géneros? y ¿cómo estas acciones contribuyen a definir la calidad de la universidad? Se propone entonces que los procesos de evaluación, tanto internos como externos, deberán necesariamente contemplar criterios de calidad con perspectiva de género, si pretenden ser procesos de reflexión críticos, serios, sistemáticos, científicos y, no solamente, ejercicios académicos para legitimar las actuales prácticas.

\section{REFERENCIAS}

Bedolla Miranda, Patricia y otros. (s.f.). Estudios de género y feminismo II. Fontana, México.

Bolaños, Carolina y Quirós, Mónica. (2004). Informe parcial. Diagnóstico Currículo inclusivo y sensible a las diferencias de género. Centro de Evaluación Académica, Universidad de Costa Rica. San José, Costa Rica. 
Bolaños Cubero, Carolina. (2003, III-IV). Currículo universitario género sensitivo e inclusivo. En: Revista de Ciencias Sociales, Universidad de Costa Rica, (101-102), 71-178.

Fernández Rius, Lourdes (2000, II). Roles de género y mujeres académicas. En: Revista de Ciencias Sociales, Universidad de Costa Rica, (88), 63-75.

Isch López, Edgar; Romero Paz, Isabel. (2001). Demandas de las mujeres universitarias. Quito, Ecuador: Centro de apoyo integral a la mujer en la Universidad Central del Ecuador.

Lagarde, Marcela. (1997). Género y Feminismo. Desarrollo humano y democracia. Madrid, España: Horas y Horas.

Universidad Central del Ecuador. (2000). Universidad y Género: palabra y testimonio. Ecuador: Universidad Central del Ecuador.

Vergés, Claude y Name, Aída. (2002). Género, currículo y ciencias de la salud. Parte III: Propuesta sobre la promoción de la equidad de género en la currícula de las ciencias de la salud. Panamá: Instituto de la mujer de la Universidad de Panamá. 